\title{
SPECTRE DES LAPLACIENS DE LICHNEROWICZ SUR LES SPHÈRES ET LES PROJECTIFS RÉELS
}

\author{
Mohamed Boucetta
}

\begin{abstract}
In this paper, we compute the spectrum of the Lichnerowicz laplacian on the symmetric forms of degree 2 on the sphere $S^{n}$ and the real projective space $\mathbb{R} P^{n}$. This is obtained by generalizing to forms the calculations of the spectrum of the laplacian on fonctions done via restriction of harmonic polynomials on euclidean space.
\end{abstract}

\section{Introduction}

Soit $(M, g)$ une variété riemannienne compacte. Pour tout $p \in \mathbb{N}$, on notera $\Omega^{p}(M)$ l'espace des $p$-formes différentielles sur $M$ et $\mathcal{S}^{p} M$ l'espace des $p$-formes symétriques sur $M$ avec $\Omega^{0}(M)=\mathcal{S}^{0} M=C^{\infty}(M)$.

Pour tout $0 \leq p \leq \operatorname{dim} M, \Omega^{p}(M)$ est muni d'un opérateur elliptique $\Delta^{p}$ à savoir le laplacien de Hodge-de Rham. dim Ker $\Delta^{p}$ étant le $p$-ième nombre de Betti de $M$, et pour d'autres propriétés, cet opérateur a été amplement étudié. Le spectre et les sous-espaces propres des $\Delta^{p}$ sur la sphère $S^{n}$, munie de sa métrique canonique, ont été déterminés dans [Be-Ga-Ma], [Be-Mi], [Ga-Me], [Ik-Ta], [Iw-Ka].

L'espace des tenseurs le plus simple à considérer, après les $\Omega^{p}(M)$, est l'espace $\mathcal{S}^{2} M$. Dans [Be-Eb], cet espace a été étudié et il a été démontré la décomposition suivante:

$\left(H_{1}\right) \quad \mathcal{S}^{2} M=\operatorname{Ker} \delta_{1} \bigoplus \delta_{1}^{*}\left(\Omega^{1}(M)\right)$, 
où $\delta_{1}^{*}: \Omega^{1}(M) \longrightarrow \mathcal{S}^{2} M$ est l'opérateur différentiel défini par

$$
\delta_{1}^{*}(\alpha)=L_{\# \alpha} g, \quad\left(\alpha \in \Omega^{1}(M)\right)
$$

$\# \alpha$ est le champ de vecteurs associée à la 1 -forme $\alpha$ grâce à la métrique $g$ et $\delta_{1}: \mathcal{S}^{2} M \longrightarrow \Omega^{1}(M)$ est l'adjoint formel de $\delta_{1}^{*}$ pour les structures préhilbertiennes sur $\Omega^{1}(M)$ et $\mathcal{S}^{2} M$ définies par la métrique $g$.

Dans le même papier, il a été démontré que Ker $\delta_{1}$ est l'espace tangent en $g$ à l'espace des structures riemaniennes sur $M$. C'est l'espace des déformations infinitésimales non-triviales de $g$.

L'espace $\mathcal{S}^{2} M$ admet aussi la décomposition (voir [Be2, p. 130])

$\left(H_{2}\right) \quad \mathcal{S}^{2} M=\operatorname{Ker} \delta_{1} \cap \operatorname{Tr}^{-1}(0) \bigoplus\left(\delta_{1}^{*}\left(\Omega^{1}(M)\right)+C^{\infty}(M) g\right)$,

où $\operatorname{Tr}: \mathcal{S}^{2} M \longrightarrow C^{\infty}(M)$ est la trace par rapport à $g$. $\operatorname{Ker} \delta_{1} \cap T r^{-1}(0)$ peut aussi être regardé comme l'espace des déformations infinitésimales non-triviales et non-conformes de $g$.

Dans [L, p. 27], Lichnerowicz a introduit, pour tout $p \in \mathbb{N}$, un laplacien $\Delta_{M}^{p}: \mathcal{S}^{p} M \longrightarrow \mathcal{S}^{p} M, \Delta_{M}^{0}$ et $\Delta_{M}^{1}$ étant les laplaciens de Hodgede Rham respectivement sur $C^{\infty}(M)$ et sur $\Omega^{1}(M)$. Les laplaciens de Lichnerowicz possédent des propriétés remarquables et se sont avérés très utiles pour l'étude de différents problèmes géométriques (voir $[\mathbf{B e}-\mathbf{E b}],[\mathbf{B e 2}],[\mathbf{M}])$. Il est à noter que le laplacien de Lichnerowicz $\Delta_{M}^{2}: \mathcal{S}^{2} M \longrightarrow \mathcal{S}^{2} M$ respecte les décompositions $\left(H_{1}\right)$ et $\left(H_{2}\right)$ si la variété $(M, g)$ est à courbure de Ricci parallèle.

Dans cet article, on se propose de calculer le spectre avec multiplicité et les sous-espaces propres de $\Delta_{S^{n}}^{2}$ sur la sphère de dimension $n$ munie de sa métrique canonique. Par suite, on exhibe deuxe bases de vecteurs propres qui engendrent deux sous-espaces propres denses dans Ker $\delta_{1}$ et dans $\operatorname{Ker} \delta_{1} \cap \operatorname{Tr}^{-1}(0)$.

Pour illustrer l'intérêt que peuvent avoir ces bases dans la résolution de versions infinitésimales de différents problèmes géométriques, on retrouve des résultats bien connus à savoir le théorème de représentation conforme sur la sphère $S^{2}[\mathbf{B e - E b}]$ et la rigidité de la structure d'Einstein canonique sur la sphère $S^{n}$. 
Finalement, en utilisant le revêtement riemannien $S^{n} \longrightarrow \mathbb{R} P^{n}$, on déduit le spectre et les sous-espaces propres de $\Delta_{\mathbb{R} P^{n}}^{1}$ et de $\Delta_{\mathbb{R} P^{n}}^{2}$.

Le calcul du spectre de $\Delta_{S^{n}}^{2}$ nécessitant la connaissance du spectre de $\Delta_{S^{n}}^{1}$, nous donnons le spectre avec multiplicité et les sous-espaces propres de $\Delta_{S^{n}}^{1}$ en utilisant une méthode qui différe légérement de celle utilisée dans [Ga-Me].

Pour ce travail, on généralise la méthode utilisée dans [Be-Ga-Ma] pour le calcul du spectre de $\Delta_{S^{n}}^{0}$ alors que les décompositions $\left(H_{1}\right)$ et $\left(H_{2}\right)$ nous servent de guides.

\section{Laplaciens de Lichnerowicz sur les tenseurs symétriques}

Soit $(M, g)$ une variété riemannienne de dimension $d$. Soit $D$ la connexion de Levi-Civita associée.

Pour tout $p \in \mathbb{N}$, le fibré vectoriel des $p$-tenseurs $\bigotimes^{p} T^{*} M \longrightarrow M$ est muni d'une structure de fibré vectoriel euclidien donnée par

$$
\langle h, f\rangle_{m}=\sum_{i_{1}, \ldots, i_{p}=1}^{d} h\left(e_{i_{1}}, \ldots, e_{i_{p}}\right) f\left(e_{i_{1}}, \ldots, e_{i_{p}}\right),
$$

où $m \in M, h, f \in \bigotimes^{p} T_{m}^{*} M$ et $\left(e_{1}, \ldots, e_{d}\right)$ est une base orthonormée quelconque de $T_{m} M$.

Pour tout entier naturel $p$, la connexion de Levi-Civita définit un opérateur différentiel $D_{p}: C^{\infty}\left(\bigotimes^{p} T^{*} M\right) \rightarrow C^{\infty}\left(\bigotimes^{p+1} T^{*} M\right)$. On notera $D_{p}^{*}$ son adjoint formel. Soit $\mathcal{S}^{p} M$ le $C^{\infty}(M)$-module des formes symétriques sur $M$.

En symétrisant l'opérateur $D$, on obtient un opérateur différentiel $\delta_{p}^{*}$ : $\mathcal{S}^{p} M \rightarrow \mathcal{S}^{p+1} M$ défini par

$$
\delta_{p}^{*} h\left(X_{1}, \ldots, X_{p+1}\right)=\sum_{i=1}^{p+1} D_{X_{i}} h\left(X_{1}, \ldots, \widehat{X}_{i}, \ldots, X_{p+1}\right) .
$$

Soit $\delta_{p}: \mathcal{S}^{p+1} M \rightarrow \mathcal{S}^{p} M$ son adjoint formel. 
L'adjoint formel de $\delta_{p}^{*}$ est appelé divergence et est donné par

$$
\delta_{p} f\left(X_{1}, \ldots, X_{p}\right)=-\sum_{i=1}^{d} D_{Y_{i}} f\left(Y_{i}, X_{1}, \ldots, X_{p}\right)
$$

où $f \in \mathcal{S}^{p+1} M,\left(X_{1}, \ldots, X_{p}\right)$ est une famille quelconque de champs de vecteurs sur $M$ et $\left(Y_{1}, \ldots, Y_{d}\right)$ est une base orthonormée de champs de vecteurs (locaux) sur $M$.

Pour tout $\alpha \in \mathcal{S}^{1} M$, on a (voir $[\mathbf{B e 2}$, p. 35])

$$
\delta_{1}^{*}(\alpha)=L_{\# \alpha} g .
$$

$\#: T^{*} M \rightarrow T M$ est l'inverse de l'homomorphisme musical $\omega^{b}: T M \rightarrow$ $T^{*} M$ qui à $v \mapsto g(v,$.$) .$

Si la variété $M$ est compacte, en tant que $\mathbb{R}$-espace vectoriel, $C^{\infty}\left(\bigotimes^{p} T^{*} M\right)$ est muni d'un produit scalaire donné par

$$
\langle h, f\rangle=\int_{M}\langle h(m), f(m)\rangle_{m} \mu_{g},
$$

où $\mu_{g}$ est la mesure canonique de $(M, g)$.

Dans le cas où $M$ est compacte, $D_{p}$ et $D_{p}^{*} ; \delta_{p}$ et $\delta_{p}^{*}$ sont adjoints pour le produit scalaire $\langle$,$\rangle .$

Définition 2.1 [L]. Le laplacien de Lichnerowicz sur les $p$-formes symétriques est l'opérateur $\Delta_{M}^{p}: \mathcal{S}^{p} M \longrightarrow \mathcal{S}^{p} M$ défini par

$$
\Delta_{M}^{p}=D_{p}^{*} D_{p}+K_{p},
$$

où $K_{p}$ est l'opérateur d'ordre 0 défini par

$$
\begin{aligned}
& K_{0}=0 \\
& K_{p}(h)\left(X_{1}, \ldots, X_{p}\right)=\sum_{i=1}^{p} r\left(X_{i}, \# i_{X_{1} \ldots \widehat{X}_{i} \ldots X_{p}} h\right) \\
& \quad-\operatorname{Tr}_{g}\left[(U, V) \mapsto \sum_{i \neq j} h\left(R\left(X_{i}, U\right) X_{j}, V, X_{1}, \ldots, \widehat{X}_{i}, \ldots, \widehat{X}_{j}, \ldots, X_{p}\right)\right],
\end{aligned}
$$

$R$ et $r$ désignent respectivement la courbure tensorielle et la courbure de Ricci de $g, \operatorname{Tr}_{g}$ désigne la trace par rapport à $g$, \# l'isomorphisme inverse de l'isomorphisme musical et $i_{X_{1} \ldots \widehat{X}_{i} \ldots X_{p}} h$ le produit intérieur de $h \operatorname{par} X_{1} \ldots \widehat{X}_{i} \ldots X_{p}$. 
Dans le cas où $M$ est compacte, $\Delta_{M}^{p}$ est un opérateur auto-adjoint pour le produit scalaire $\langle$,$\rangle .$

\section{Remarques.}

i) $\Delta_{M}^{0}$ et $\Delta_{M}^{1}$ sont les laplaciens de Hodge-de Rham respectivement sur $C^{\infty}(M)$ et $\mathcal{S}^{1} M$. La formule

$$
\Delta_{M}^{1}=D_{1}^{*} D_{1}+K_{1}
$$

n'est rien d'autre que la fameuse formule de Bochner [Boc], puisque $K_{1}(\alpha)=r(\# \alpha,$.$) , pour tout \alpha \in \mathcal{S}^{1} M$.

ii) On a clairement

$$
\Delta_{M}^{1} \circ d=d \circ \Delta_{M}^{0}
$$

Théorème 2.1. Les définitions et notations sont celles ci-dessus. On a les propriétés suivantes:

i) Si la métrique g est à courbure de Ricci parallèle, on a

$$
\Delta_{M}^{2} \circ \delta_{1}^{*}=\delta_{1}^{*} \circ \Delta_{M}^{1} \quad \text { et } \quad \Delta_{M}^{1} \circ \delta_{1}=\delta_{1} \circ \Delta_{M}^{2} .
$$

ii) $\operatorname{Tr}_{g} \circ \Delta_{M}^{2}=\Delta_{M}^{0} \circ T r_{g}$.

iii) Pour toute fonction $f \in C^{\infty}(M)$, on a $\Delta_{M}^{2}(f g)=\Delta_{M}^{0}(f) g$.

Preuve: Pour i) et ii) voir [L, pp. 28-29]. Montrons, maintenant iii). Soit $h \in \mathcal{S}^{2} M$ quelconque. On a

$$
\left\langle\Delta_{M}^{2}(f g), h\right\rangle=\left\langle f g, \Delta_{M}^{2}(h)\right\rangle=\int_{M} f \operatorname{Tr}_{g}\left(\Delta_{M}^{2}(h) \mu_{g} .\right.
$$

En utilisant ii), on obtient

$$
\left\langle\Delta_{M}^{2}(f g), h\right\rangle=\int_{M} f \Delta_{M}^{0}\left(T r_{g} h\right) \mu_{g}=\int_{M} \operatorname{Tr}_{g} h \Delta_{M}^{0}(f) \mu_{g}=\left\langle h, \Delta_{M}^{0}(f) g\right\rangle .
$$

D'où le résultat. 
Lemme 2.1. Pour tout entier $p>0$ et pour tout $h \in \mathcal{S}^{p} M$, on a

$$
\delta_{p} \circ \delta_{p}^{*} h-\delta_{p-1}^{*} \circ \delta_{p-1} h=D_{p}^{*} D_{p} h-K_{p}(h) .
$$

Preuve: Pour établir cette égalité, il suffit de la vérifier pour les formes pôlaires. Soit $X$ un champs de vecteurs et soit $\left(E_{1}, \ldots, E_{d}\right)$ une base orthonormée de champs de vecteurs (locaux). On a

$$
\begin{aligned}
& \delta_{p} \circ \delta_{p}^{*} h(X, \ldots, X) \\
& =-\sum_{i=1}^{d} D_{E_{i}} \delta_{p}^{*} h\left(E_{i}, X, \ldots, X\right) \\
& =-\sum_{i=1}^{d} E_{i} \cdot \delta_{p}^{*} h\left(E_{i}, X, \ldots, X\right)+\sum_{i=1}^{d} \delta_{p}^{*} h\left(D_{E_{i}} E_{i}, X, \ldots, X\right) \\
& +p \sum_{i=1}^{d} \delta_{p}^{*} h\left(E_{i}, D_{E_{i}} X, X, \ldots, X\right) \\
& =-\sum_{i=1}^{d} E_{i} \cdot D_{E_{i}} h(X, \ldots, X)-p \sum_{i=1}^{d} E_{i} . D_{X} h\left(E_{i}, X, \ldots, X\right) \\
& +\sum_{i=1}^{d} D_{D_{E_{i}} E_{i}} h(X, \ldots, X)+p \sum_{i=1}^{d} D_{X} h\left(D_{E_{i}} E_{i}, X, \ldots, X\right) \\
& +p \sum_{i=1}^{d} D_{E_{i}} h\left(D_{E_{i}} X, X, \ldots, X\right)+p \sum_{i=1}^{d} D_{D_{E_{i}} X} h\left(E_{i}, X, \ldots, X\right) \\
& +p(p-1) \sum_{i=1}^{d} D_{X} h\left(E_{i}, D_{E_{i}} X, X, \ldots, X\right) \\
& =-\sum_{i=1}^{d} E_{i} . D_{E_{i}} h(X, \ldots, X)-p \sum_{i=1}^{d} D_{E_{i}} D_{X} h\left(E_{i}, X, \ldots, X\right) \\
& +\sum_{i=1}^{d} D_{D_{E_{i}} E_{i}} h(X, \ldots, X)+p \sum_{i=1}^{d} D_{E_{i}} h\left(D_{E_{i}} X, X, \ldots, X\right) \\
& p \sum_{i=1}^{d} D_{D_{E_{i}} X} h\left(E_{i}, X, \ldots, X\right) \\
& =D_{p}^{*} D_{p} h(X, \ldots, X)-p \sum_{i=1}^{d} D_{\left(E_{i}, X\right)}^{2} h\left(E_{i}, X, \ldots, X\right) .
\end{aligned}
$$


D'un autre côté et par un calcul direct, on obtient

$$
\begin{aligned}
\delta_{p-1}^{*} \circ \delta_{p-1} h(X, \ldots, X)= & -p \sum_{i=1}^{d} D_{\left(X, E_{i}\right)}^{2} h\left(E_{i}, X, \ldots, X\right) \\
& -p \sum_{i=1}^{d}\left(D_{E_{i}} h\left(D_{X} E_{i}, X, \ldots, X\right)\right. \\
& \left.+D_{D_{X} E_{i}} h\left(E_{i}, X, \ldots, X\right)\right) .
\end{aligned}
$$

En utilisant l'identité de Ricci, on obtient donc

$$
\begin{aligned}
\delta_{p} \circ \delta_{p}^{*} h(X, \ldots, X)-\delta_{p-1}^{*} \circ \delta_{p-1} h(X, \ldots, X)=D_{p}^{*} D_{p} h(X, \ldots, X) \\
\quad-\left[p r\left(X, \#\left(i_{X \ldots X}^{p-1} h\right)\right)-p(p-1) \sum_{i=1}^{d} h\left(R\left(E_{i}, X\right) X, E_{i}, X, \ldots, X\right)\right] \\
\quad+p \sum_{i=1}^{d}\left(D_{E_{i}} h\left(D_{X} E_{i}, X, \ldots, X\right)+D_{D_{X} E_{i}} h\left(E_{i}, X, \ldots, X\right)\right) .
\end{aligned}
$$

Or, pour tout $v$ vecteur tangent en un point $m$, il existe un champ de vecteurs $X$ et une base orthonormée $\left(E_{i}, \ldots, E_{d}\right)$ tels que $X(m)=v$ et $\left(D_{X} E_{i}\right)_{m}=0$ pour $i=1, \ldots, d$. Comme c'est une relation tensorielle, on a le lemme.

Remarque importante. En vertu ce lemme, on obtient une expression plus fine du laplacien de Lichnerowicz à savoir

$$
\Delta_{M}^{p}=\delta_{p} \circ \delta_{p}^{*}-\delta_{p-1}^{*} \circ \delta_{p-1}+2 K_{p} .
$$

Cette expression nous sera très utile par la suite.

Proposition 2.1. Soit $h=\sum_{i_{1}, \ldots, i_{p}=1}^{d} h_{i_{1}, \ldots, i_{p}} d x_{i_{1}} \ldots d x_{i_{p}}$ une $p$-forme symétrique sur $\left(\mathbb{R}^{d}\right.$, can $)$. On a

$$
\Delta_{\mathbb{R}^{d}}^{p} h=\sum_{i_{1}, \ldots, i_{p}=1}^{d} \Delta_{0} h_{i_{1}, \ldots, i_{p}} d x_{i_{1}} \ldots d x_{i_{p}}
$$

avec $\Delta_{0}=-\sum_{i=1}^{d} \frac{\partial^{2}}{\partial x_{i}^{2}}$.

Preuve: Evidente. 


\section{Laplaciens de Lichnerowicz sur les sphères}

\subsection{Préliminaires.}

On se place maintenant dans $\mathbb{R}^{n+1}$ muni de sa métrique canonique qu'on notera indifféremment can ou $\langle$,$\rangle . On notera \widetilde{D}$ la connexion de Levi-Civita associée, $\vec{r}$ le champ de vecteurs radial et $N=\frac{\partial}{\partial r}$ le champ de vecteurs unitaire radial.

$$
\vec{r}=\sum_{i=1}^{n+1} x_{i} \frac{\partial}{\partial x_{i}}, \quad N=\frac{1}{r} \sum_{i=1}^{n+1} x_{i} \frac{\partial}{\partial x_{i}}
$$

avec $r=\sqrt{x_{1}^{2}+\ldots+x_{n+1}^{2}}$ et $\left(x_{1}, \ldots, x_{n+1}\right)$ les coordonnées canoniques de $\mathbb{R}^{n+1}$.

Pour tout champ de vecteurs $X$ sur $\mathbb{R}^{n+1}$, on a

$$
\widetilde{D}_{X} N=\frac{1}{r}(X-\langle X, N\rangle N)
$$

En particulier $\widetilde{D}_{N} N=0$.

Soit $D$ la connexion de Levi-Civita associée à la métrique canonique de $S^{n}$. Pour tout champs de vecteurs $X, Y$ tangents à $S^{n}$, on a

$$
\widetilde{D}_{X} Y=D_{X} Y-\langle X, Y\rangle N \text {. }
$$

Proposition 3.1. Soit $H \in \mathcal{S}^{p} \mathbb{R}^{n+1}$ et soit $\left(X, X_{1}, \ldots, X_{p}\right)$ une famille de champs de vecteurs tangents à $S^{n}$. Soit h la restriction de $H$ $\grave{a} S^{n}$. Alors, en restriction à $S^{n}$, les formules suivantes sont vérifiées:

$$
\begin{aligned}
\widetilde{D}_{X} H\left(X_{1}, \ldots, X_{p}\right)= & D_{X} h\left(X_{1}, \ldots, X_{p}\right) \\
& +\sum_{i=1}^{p}\left\langle X, X_{i}\right\rangle H\left(N, X_{1}, \ldots, \widetilde{X}_{i}, \ldots, X_{p}\right), \\
\widetilde{D}_{N} H\left(X_{1}, \ldots, X_{p}\right)= & L_{N} H\left(X_{1}, \ldots, X_{p}\right)-p h\left(X_{1}, \ldots, X_{p}\right), \\
\widetilde{D}_{N} H\left(N, X_{1}, \ldots, X_{p-1}\right)= & L_{N} \circ i_{N} H\left(X_{1}, \ldots, X_{p-1}\right) \\
& -(p-1) H\left(N, X_{1}, \ldots, X_{p-1}\right) .
\end{aligned}
$$


Preuve: La première formule est une conséquence immédiate de (3).

On a

$\widetilde{D}_{N} H\left(X_{1}, \ldots, X_{p}\right)=N \cdot H\left(X_{1}, \ldots, X_{p}\right)-\sum_{i=1}^{p} H\left(X_{1}, \ldots, \widetilde{D}_{N} X_{i}, \ldots, X_{p}\right)$.

Or $\widetilde{D}_{N} X_{i}=\left[N, X_{i}\right]+\widetilde{D}_{X_{i}} N$ et, en restriction à $S^{n}$, on a en vertu de (3), $\widetilde{D}_{X_{i}} N=X_{i}$. Ceci permet d'établir la deuxième formule.

Un calcul analogue donnerait la troisième formule.

Dans tout ce qui suit, on notera $\widetilde{\delta}^{*}$ et $\widetilde{\delta}$ respectivement la codivergence et la divergence de $\left(\mathbb{R}^{n+1}\right.$, can $)$ et $\delta^{*}$ et $\delta$ ceux de $\left(S^{n}\right.$, can $)$.

Proposition 3.2. Soit $H \in \mathcal{S}^{p+1} \mathbb{R}^{n+1}$ et soit $h$ sa restriction à $S^{n}$. Alors, la formule suivante est vérifiée en restriction à $S^{n}$ :

$$
\widetilde{\delta}_{p} H=\delta_{p} h-n i_{N} H-L_{N} \circ i_{N} H .
$$

Preuve: Soit $x \in S^{n}$ et soit $\left(E_{1}, \ldots, E_{n}\right)$ une base orthonormée de champs de vecteurs au voisinage de $x$ et tangents en $x$ à $S^{n}$. Soit $\left(X_{1}, \ldots, X_{p}\right)$ une famille de champs de vecteurs tangents à $S^{n}$. On a

$\widetilde{\delta}_{p} H\left(X_{1}, \ldots, X_{p}\right)=-\sum_{i=1}^{n} \widetilde{D}_{E_{i}} H\left(E_{i}, X_{1}, \ldots, X_{p}\right)-\widetilde{D}_{N} H\left(N, X_{1}, \ldots, X_{p}\right)$.

Or, d'après la Proposition 2.1, on a

$$
\begin{aligned}
\widetilde{D}_{E_{i}} H\left(E_{i}, X_{1}, \ldots, X_{p}\right)= & D_{E_{i}} h\left(E_{i}, X_{1}, \ldots, X_{p}\right) \\
& +\left\langle E_{i}, E_{i}\right\rangle H\left(N, X_{1}, \ldots, X_{p}\right) \\
& +\sum_{j=1}^{p} H\left(N,\left\langle E_{i}, X_{j}\right\rangle E_{i}, X_{1}, \ldots, \widetilde{X}_{j}, \ldots, X_{p}\right), \\
\widetilde{D}_{N} H\left(N, X_{1}, \ldots, X_{p}\right)= & L_{N} \circ i_{N} H\left(X_{1}, \ldots, X_{p}\right)-p H\left(N, X_{1}, \ldots, X_{p}\right) .
\end{aligned}
$$

Ceci permet de conclure.

On pourrait, de la même manière, établir une formule reliant $\widetilde{\delta}_{p}^{*}$ et $\delta_{p}^{*}$; mais pour ce qu'on envisage de faire, on se contentera d'une formule dans les cas $p=0,1,2$. 
Proposition 3.3. Soient $\widetilde{\alpha} \in \mathcal{S}^{1} \mathbb{R}^{n+1}$ et $H \in \mathcal{S}^{2} \mathbb{R}^{n+1}$. Soient $\alpha$ et $h$ leurs restrictions à $S^{n}$. En restriction $\grave{a} S^{n}$, on a

$$
\begin{aligned}
\widetilde{\delta}_{1}^{*} \widetilde{\alpha} & =\delta_{1}^{*} \alpha+2 \widetilde{\alpha}(N) \text { can, } \\
\widetilde{\delta}_{2}^{*} H & =\delta_{2}^{*} h+2 R(H),
\end{aligned}
$$

avec $R(H)(X, Y, Z)=H(N,\langle X, Y\rangle Z+\langle Y, Z\rangle X+\langle Z, X\rangle Y)$.

Preuve: Ces deux formules sont une conséquence immédiate de la Proposition 3.1.

3.2. Formule reliant $\Delta_{\mathbb{R}^{n+1}}^{0}$ et $\Delta_{S^{n}}^{0}$.

Proposition 3.4. Soit $F$ une fonction sur $R^{n+1}$ et soit $f$ sa restriction à $S^{n}$. En restriction à $S^{n}$, on a

$$
\Delta_{\mathbb{R}^{n+1}}^{0} F=\Delta_{S^{n}}^{0} f-n \frac{\partial F}{\partial r}-\frac{\partial^{2} F}{\partial r^{2}} .
$$

Preuve: On remarque que $\Delta_{\mathbb{R}^{n+1}}^{0} F=\widetilde{\delta}_{0} d F$ et on applique la Proposition 3.2 pour $p=0$.

3.3. Formule reliant $\Delta_{\mathbb{R}^{n+1}}^{1}$ et $\Delta_{S^{n}}^{1}$.

En vertu de (2) et du fait que $\left(S^{n}\right.$, can) est une variété d'Einstein dont la courbure de Ricci $r_{\text {can }}=(n-1)$ can, on a

$$
\Delta_{S^{n}}^{1}=\delta_{1} \circ \delta_{1}^{*}-\delta_{0}^{*} \circ \delta_{0}+2(n-1) \mathrm{Id} .
$$

Pour établir une relation entre $\Delta_{\mathbb{R}^{n+1}}^{1}$ et $\Delta_{S^{n}}^{1}$, on aura besoin des deux formules suivantes:

Soit $\alpha \in \mathcal{S}^{1} \mathbb{R}^{n+1}$, on a

$$
i_{N} \widetilde{\delta}_{1}^{*} \alpha=\widetilde{\delta}_{0}^{*} i_{N} \alpha+L_{N} \alpha-\frac{2}{r} \alpha+\frac{2}{r} \alpha(N) i_{N} \operatorname{can} .
$$

Soit $f \in C^{\infty}\left(S^{n}\right)$, on a

$$
\delta_{1}(f \operatorname{can})=-d f .
$$

Etablissons la formule (6). Soit $X$ un champ de vecteurs sur $\mathbb{R}^{n+1}$.

$$
\begin{aligned}
i_{N} \widetilde{\delta}_{1}^{*} \alpha(X) & =X . \alpha(N)+N \cdot \alpha(X)-\alpha\left(\widetilde{D}_{N} X\right)-\alpha\left(\widetilde{D}_{X} N\right) \\
& =\widetilde{\delta}_{0}^{*} i_{N} \alpha(X)+N \cdot \alpha(X)-\alpha([N, X])-2 \alpha\left(\widetilde{D}_{X} N\right) .
\end{aligned}
$$

En appliquant (3), on obtient (6). Un calcul direct donnerait la formule (7). 
Proposition 3.5. Soit $\widetilde{\alpha} \in \mathcal{S}^{1} \mathbb{R}^{n+1}$ et $\alpha$ sa restriction $\grave{a} S^{n}$. En restriction à $S^{n}$, on a

$$
\Delta_{\mathbb{R}^{n+1}}^{1} \widetilde{\alpha}=\Delta_{S^{n}}^{1} \alpha-L_{N} \circ L_{N} \widetilde{\alpha}-(n-2) L_{N} \widetilde{\alpha}-2 d \widetilde{\alpha}(N) .
$$

Preuve: D'après les Propositions 3.2 et 3.3 , on a

$$
\begin{aligned}
\widetilde{\delta}_{1} \widetilde{\delta}_{1}^{*} \widetilde{\alpha} & =\delta_{1} \widetilde{\delta}_{1}^{*} \widetilde{\alpha}-n i_{N} \widetilde{\delta}_{1}^{*} \widetilde{\alpha}-L_{N} i_{N} \widetilde{\delta}_{1}^{*} \widetilde{\alpha} \\
& =\delta_{1} \delta_{1}^{*} \alpha+2 \delta_{1}(\widetilde{\alpha}(N) \operatorname{can})-n i_{N} \widetilde{\delta}_{1}^{*} \widetilde{\alpha}-L_{N} i_{N} \widetilde{\delta}_{1}^{*} \widetilde{\alpha}
\end{aligned}
$$

D'après la formule (6)

$$
\begin{aligned}
L_{N} \circ i_{N} \widetilde{\delta}_{1}^{*} \widetilde{\alpha}=L_{N} \widetilde{\delta}_{0}^{*} i_{N} \widetilde{\alpha} & +L_{N} \circ L_{N} \widetilde{\alpha}+\frac{2}{r^{2}} \widetilde{\alpha}-\frac{2}{r} L_{N} \widetilde{\alpha} \\
& +N\left(\frac{2}{r} \widetilde{\alpha}(N)\right) i_{N} \operatorname{can}+\frac{2}{r} \widetilde{\alpha}(N) L_{N} \circ i_{N} \operatorname{can} .
\end{aligned}
$$

Puisque, en restriction à $S^{n}, L_{N} i_{N}$ can $=0$ et $i_{N}$ can $=0$, on obtient donc, en utilisant (7) et toujours en restriction à $S^{n}$,

$$
\begin{aligned}
\widetilde{\delta}_{1} \widetilde{\delta}_{1}^{*} \widetilde{\alpha}=\delta_{1} \delta_{1}^{*} \alpha-2 d \widetilde{\alpha}(N)-L_{N} \circ L_{N} \widetilde{\alpha}-(n-2) L_{N} \widetilde{\alpha} \\
+2(n-1) \alpha-n \widetilde{\delta}_{0}^{*} i_{N} \widetilde{\alpha}-L_{N} \widetilde{\delta}_{0}^{*} i_{N} \widetilde{\alpha} .
\end{aligned}
$$

D'un autre côté, toujours en vertu de la Proposition 3.2,

$$
\widetilde{\delta}_{0}^{*} \widetilde{\delta}_{0} \widetilde{\alpha}=\delta_{0}^{*} \delta_{0} \alpha-n d \widetilde{\alpha}(N)-d L_{N} i_{N} \widetilde{\alpha} .
$$

Ceci permet de conclure, en remarquant que $\widetilde{\delta}_{0}^{*}=d$ et que celle ci commute avec la restriction à $S^{n}$ et la dérivée de Lie.

3.4. Formule reliant $\Delta_{\mathbb{R}^{n+1}}^{2}$ et $\Delta_{S^{n}}^{2}$.

Proposition 3.6. On a

$$
\Delta_{S^{n}}^{2} h=\delta_{2} \circ \delta_{2}^{*} h-\delta_{1}^{*} \circ \delta_{1} h+4(n h-T r h \text { can }) .
$$

Preuve: C'est une conséquence de (2) et du fait que

$$
K_{2}(h)=2(n h-T r h \text { can }) \text {. }
$$


Cette formule découle des deux formules suivantes:

$$
\begin{aligned}
r(X, Y) & =(n-1) \operatorname{can}(X, Y), \\
R(X, Y) Z & =\operatorname{can}(Y, Z) X-\operatorname{can}(X, Z) Y .
\end{aligned}
$$

$R, r$ les courbures tensorielle et de Ricci sur $\left(S^{n}\right.$, can $)$ et $(X, Y, Z)$ un triple de champs de vecteurs quelconques sur $S^{n}$.

Pour trouver une formule reliant $\Delta_{\mathbb{R}^{n+1}}^{2}$ et $\Delta_{S^{n}}^{2}$, le calcul sera identique au calcul fait dans le cas $p=1$. On aura besoin de calculer $\delta_{2}(R(H))$ où $R(H)$ est la 3 -forme définie dans la Proposition 3.3 et d'établir une relation entre $i_{N} \widetilde{\delta}_{2}^{*}$ et $\widetilde{\delta}_{1}^{*} i_{N}$. En plus, contrairement au cas précédent, la dérivée de Lie ne commute pas avec $\widetilde{\delta}_{1}^{*}$.

Soit $H \in \mathcal{S}^{2} \mathbb{R}^{n+1}$ et soit $R(H)$ la 3 -forme définie dans la Proposition 3.3. On notera aussi $R(H)$ sa restriction à $S^{n}$.

Un clacul direct donnerait

$$
\begin{gathered}
\delta_{2}(R(H))=-\delta_{1}^{*}\left(i_{N} H\right)+\delta_{0}\left(i_{N} H\right) \text { can } \\
i_{N} \widetilde{\delta}_{2}^{*} H=\widetilde{\delta}_{1}^{*} i_{N} H+L_{N} H-\frac{4}{r} H+\frac{4}{r} i_{N} H \odot i_{N} \text { can } .
\end{gathered}
$$

$\odot$ désigne le produit symétrique.

Lemme 3.1. Soit $(M, g)$ une variété riemannienne, soit $D$ sa connexion de Levi-Civita et soit $R$ son tenseur de courbure. Soit $\alpha \in \mathcal{S}^{1} M$. Pour tout champs de vecteurs $X, Y, N$ de $M$, on a la formule

$$
L_{N} \delta_{1}^{*} \alpha(X, Y)=\delta_{1}^{*} L_{N} \alpha(X, Y)+\alpha\left(R^{s}(N, X, Y)-D_{X, Y}^{2, s} N\right)
$$

avec

$$
\begin{aligned}
R^{s}(N, X, Y) & =R(N, X, Y)+R(N, Y, X), \\
D_{X, Y}^{2, s} N & =D_{X} D_{Y} N+D_{Y} D_{X} N-D_{D_{X} Y} N-D_{D_{Y} X} N .
\end{aligned}
$$

Preuve du lemme: Un calcul direct et fastidieux.

Soient $X, Y$ deux champs de vecteurs tangents à $S^{n}$. En restriction à $S^{n}$, on a

$$
D_{X, Y}^{2, s} N=-2\langle X, Y\rangle N .
$$


Proposition 3.7. Soit $H \in \mathcal{S}^{2} \mathbb{R}^{n+1}$ et soit $h$ sa restriction à $S^{n}$. On a, en restriction $\grave{a} S^{n}$,

$$
\begin{aligned}
\Delta_{\mathbb{R}^{n+1}}^{2} H=\Delta_{S^{n}}^{2} h-L_{N} \circ & L_{N} H-(n-4) L_{N} H-4 h \\
& -2 \delta_{1}^{*}\left(i_{N} H\right)-2 H(N, N) \text { can }+2 T r h \text { can } .
\end{aligned}
$$

Preuve: D'après les Propositions 3.2 et 3.3 , on a

$$
\widetilde{\delta}_{2} \widetilde{\delta}_{2}^{*} H=\delta_{2} \delta_{2}^{*} h+2 \delta_{2}(R(H))-n i_{N} \widetilde{\delta}_{2}^{*} H-L_{N} i_{N} \widetilde{\delta}_{2}^{*} H
$$

En utilisant la formule (10), on obtient,

$$
\begin{aligned}
L_{N} i_{N} \widetilde{\delta}_{2}^{*} H=L_{N} \widetilde{\delta}_{1}^{*} i_{N} H+L_{N} o L_{N} H+\frac{4}{r^{2}} & H-\frac{4}{r} L_{N} H \\
& +L_{N}\left(\frac{4}{r} i_{N} H \odot i_{N} \text { can }\right) .
\end{aligned}
$$

D'après le Lemme 3.2 et la formule (11), on a

$$
L_{N} \widetilde{\delta}_{1}^{*} i_{N} H=\widetilde{\delta}_{1}^{*} L_{N} i_{N} H+2 H(N, N) \operatorname{can}
$$

On obtient, donc, en utilisant (9),

$$
\begin{aligned}
\widetilde{\delta}_{2} \widetilde{\delta}_{2}^{*} H= & \delta_{2} \delta_{2}^{*} h-L_{N} \circ L_{N} H-(n-4) L_{N} H+4(n-1) H \\
& -n \widetilde{\delta}_{1}^{*} i_{N} H-\widetilde{\delta}_{1}^{*} L_{N} i_{N} H+2 \delta_{0}\left(i_{N} H\right) \\
& -2 \delta_{1}^{*}\left(i_{N} H\right)-2 H(N, N) \operatorname{can} .
\end{aligned}
$$

D'un autre côté, en vertu des Propositions 3.2 et 3.3 , on a

$$
\widetilde{\delta}_{1}^{*} \widetilde{\delta}_{1} H=\delta_{1}^{*} \delta_{1} h-n \delta_{1}^{*}\left(i_{n} H\right)-\delta_{1}^{*}\left(L_{N} i_{N} H\right)+2 \widetilde{\delta}_{1} H(N) \text { can }
$$

Or, d'après la Proposition 3.3, on a

$$
\begin{aligned}
\delta_{1}^{*}\left(i_{N} H\right) & =\widetilde{\delta}_{1}^{*}\left(i_{N} H\right)-2 H(N, N) \text { can } \\
\delta_{1}^{*}\left(L_{N} i_{N} H\right) & =\widetilde{\delta}_{1}^{*}\left(L_{N} i_{N} H\right)-2 N \cdot H(N, N) \text { can } .
\end{aligned}
$$

Un calcul direct donnerait

$$
\widetilde{\delta}_{1} H(N)=\delta_{0}\left(i_{N} H\right)-n H(N, N)-N \cdot H(N, N)+T r h .
$$


Finalement, on a

$$
\widetilde{\delta}_{1}^{*} \widetilde{\delta}_{1} H=\delta_{1}^{*} \delta_{1} h-n \widetilde{\delta}_{1}^{*}\left(i_{n} H\right)-\widetilde{\delta}_{1}^{*}\left(L_{N} i_{N} H\right)+2\left(\delta_{0}\left(i_{N} H\right)+T r h\right) \text { can } .
$$

Ceci permet de conclure.

Dans tout ce qui suit, on notera:

$\widetilde{P}_{k}$ l'espace vectoriel des polynômes homogènes de degré $k$ sur $\mathbb{R}^{n+1}$.

$\widetilde{H}_{k}$ l'espace vectoriel des polynômes homogènes et harmoniques de degré $k$ sur $\mathbb{R}^{n+1}$.

$\mathcal{S}^{p} \widetilde{P}_{k}$ (resp. $\mathcal{S}^{p} \widetilde{H}_{k}$ ) l'espace vectoriel des $p$-formes symétriques sur $\mathbb{R}^{n+1}$ dont toutes les composantes, dans la base canonique, sont dans $\widetilde{P}_{k}\left(\right.$ resp. dans $\left.\widetilde{H}_{k}\right)$.

Pour toute $p$-forme symétrique sur $\mathbb{R}^{n+1}, h=\sum_{i_{1}, \ldots, i_{p}=1}^{n+1} h_{i_{1}, \ldots, i_{p}} d x_{i_{1}} \ldots d x_{i_{p}}$, on pose

$$
N . h=\sum_{i_{1}, \ldots, i_{p}=1}^{n+1} N \cdot h_{i_{1}, \ldots, i_{p}} d x_{i_{1}} \ldots d x_{i_{p}} .
$$

La propriété ii) de la proposition suivante va jouer un rôle très important par la suite.

Proposition 3.8. i) Pour tout $Q \in \widetilde{P}_{k}$, et tout $s$ réel, on a

$$
\Delta_{\mathbb{R}^{n+1}}^{0} r^{s} Q=r^{s} \Delta_{\mathbb{R}^{n+1}}^{0} Q-s(s+n-1+2 \operatorname{deg} Q) r^{s-2} Q .
$$

ii) Soit $Q \in \widetilde{H}_{k}$. Pour tout $j=1 \ldots n+1$, il existe un couple unique $\left(Q_{0}, Q_{1}\right)$ de polynômes homogènes harmoniques tels que

$$
x_{j} Q=Q_{0}+r^{2} Q_{1} .
$$

Plus précisement, on a $Q_{0} \in \widetilde{H}_{k+1}$ et $Q_{1} \in \widetilde{H}_{k-1}$.

Preuve: i) C'est un calcul direct.

ii) Il suffit de prendre

$$
\begin{aligned}
Q_{0} & =x_{j} Q-\frac{1}{2 k+n-1} r^{2} \frac{\partial Q}{\partial x_{j}}, \\
Q_{1} & =\frac{1}{2 k+n-1} \frac{\partial Q}{\partial x_{j}}
\end{aligned}
$$

et d'appliquer i) pour vérifier que $Q_{0}$ est harmonique. 


\subsection{Spectre et sous-espaces propres de $\Delta_{S^{n}}^{0}$.}

Proposition 3.9. Soit $f$ la restriction à $S^{n}$ d'un élément de $\widetilde{H}_{k}$. On

$$
\Delta_{S^{n}}^{0} f=k(k-1+n) f .
$$

En plus, la multiplicité de la valeur propre est égale à la dimension de $\widetilde{H}_{k}$ qui est égale à

$$
\frac{n(n+1) \ldots(n+k-3)(n+k-2)}{k !}(n+2 k-1) .
$$

Preuve: Découle immédiatement de la Proposition 3.4. Pour la multiplicité voir [Be-Ga-Ma, p. 162].

Cette proposition permet d'avoir le spectre et tous les sous-espace propres de $\Delta_{S^{n}}^{0}$ puisque les fonctions polynômes sont denses dans $C^{0}\left(S^{n}\right)$ et on a la décomposition orhogonale ([Be-Ga-Ma, p. 160])

$$
\widetilde{P}_{k}=\bigoplus_{l=0}^{\left[\frac{k}{2}\right]} r^{2 l} \widetilde{H}_{k-2 l} .
$$

3.6. Spectre et sous-espaces propres de $\Delta_{S^{n}}^{1}$.

Il est connu, d'après un théorème de Hodge et puisque $\operatorname{Ker} \Delta_{S^{n}}^{1}=0$, que

$$
\left(H_{3}\right) \quad \mathcal{S}^{1} S^{n}=d C^{\infty}\left(S^{n}\right) \bigoplus \operatorname{Ker} \delta_{0} .
$$

Cette décomposition est orthogonale et invariante par $\Delta_{S^{n}}^{1}$.

Proposition 3.10. Soit $\alpha \in \mathcal{S}^{1} \mathbb{R}^{n+1}$. On a

$$
\begin{aligned}
L_{N} \alpha & =N . \alpha+\frac{1}{r} \alpha-\frac{1}{r} \alpha(N) i_{N} \text { can, } \\
L_{N} \circ L_{N} \alpha & =N . N . \alpha+\frac{2}{r} N . \alpha-\frac{2}{r} N . \alpha(N) i_{N} \text { can } .
\end{aligned}
$$

Preuve:

$$
\begin{aligned}
L_{N} \alpha\left(\frac{\partial}{\partial x_{i}}\right) & =N \cdot \alpha\left(\frac{\partial}{\partial x_{i}}\right)-\alpha\left(\left[N, \frac{\partial}{\partial x_{i}}\right]\right) \\
& =N \cdot \alpha_{i}-\alpha\left(\widetilde{D}_{N} \frac{\partial}{\partial x_{i}}\right)+\alpha\left(\widetilde{D}_{\frac{\partial}{\partial x_{i}}} N\right) \\
& =N \cdot \alpha_{i}+\frac{1}{r}\left(\alpha_{i}-\left\langle N, \frac{\partial}{\partial x_{i}}\right\rangle \alpha(N)\right),
\end{aligned}
$$

et ce en vertu de (3).

La deuxième formule est une application de la première. 
Proposition 3.11. Soit $\widetilde{\alpha} \in \mathcal{S}^{1} \widetilde{P}_{k}$ et soit $\alpha$ sa restriction à $S^{n}$. On a, en restriction $\grave{a} S^{n}$,

$$
\Delta_{\mathbb{R}^{n+1}}^{1} \widetilde{\alpha}=\Delta_{S^{n}}^{1} \alpha-(k(k+n-1)+n-2) \alpha-2 d \widetilde{\alpha}(\vec{r}) .
$$

Preuve: D'après la Proposition 3.10, on a, en restriction à $S^{n}$,

$$
L_{N} \widetilde{\alpha}=(k+1) \widetilde{\alpha} \quad \text { et } \quad L_{N} \circ L_{N} \widetilde{\alpha}=k(k+1) \widetilde{\alpha} .
$$

La proposition découle alors de la Proposition 3.5 et du fait, qu'en restriction à $S^{n}, d \widetilde{\alpha}(N)=d \widetilde{\alpha}(\vec{r})$.

Proposition 3.12. Soit $f$ la restriction à $S^{n}$ d'un élément de $\widetilde{H}_{k}$. On a

$$
\Delta_{S^{n}}^{1} d f=k(k+n-1) d f
$$

Preuve: Découle de la Proposition 3.9 et du fait que $\Delta_{S^{n}}^{1}$ et $\Delta_{S^{n}}^{0}$ commutent avec $d$.

D'après $\left(H_{3}\right)$, cette proposition nous donne le spectre et les sousespaces propres de $\Delta_{S^{n}}^{1}$ restreint à $d C^{\infty}\left(S^{n}\right)$. On va, dans ce qui suit, donner le spectre et les sous-espaces propres de $\Delta_{S^{n}}^{1}$ restreint à $\operatorname{Ker} \delta_{0}$.

Soit $\widetilde{\alpha} \in \mathcal{S}^{1} \widetilde{H}_{k}$. Contrairement au cas du $\Delta_{S^{n}}^{0}$, la restriction $\alpha$ de $\widetilde{\alpha}$ à $S^{n}$ n'est pas un vecteur propre de $\Delta_{S^{n}}^{1}$. Dans ce qui suit, on va donner la décomposition de $\alpha$ suivant $\left(H_{3}\right)$.

On a, d'après la Proposition 3.8 ii),

$$
\widetilde{\alpha}(\vec{r})=\sum_{i=1}^{n+1} \alpha_{i} x_{i}=Q_{0}+r^{2} Q_{1} \quad \text { avec } \quad Q_{l} \in \widetilde{H}_{k+1-2 l} .
$$

On pose

$$
\omega_{k}(\widetilde{\alpha})=\widetilde{\alpha}-\frac{1}{k+1} d Q_{0}-\frac{1}{2-k-n} d r^{2} Q_{1} .
$$

$\omega_{k}(\widetilde{\alpha}) \in \mathcal{S}^{1} \widetilde{P}_{k}$ et on notera $\omega_{k}(\alpha)$ sa restriction à $S^{n}$.

Proposition 3.13. On a

$$
\Delta_{S^{n}}^{1} \omega_{k}(\alpha)=(k(k+n-1)+n-2) \omega_{k}(\alpha) .
$$


Preuve: D'après (13), on a

$$
\begin{aligned}
\omega_{k}(\widetilde{\alpha})(\vec{r}) & =\frac{1-2 k-n}{2-k-n} r^{2} Q_{1} \\
\Delta_{\mathbb{R}^{n+1}}^{1} \omega_{k}(\widetilde{\alpha}) & =-\frac{1}{2-k-n} d \Delta_{\mathbb{R}^{n+1}}^{0}\left(r^{2} Q_{1}\right) \\
& =-2 \frac{1-2 k-n}{2-k-n} d Q_{1},
\end{aligned}
$$

d'après i) Proposition 2.8. On aura donc

$$
2 d \omega_{k}(\widetilde{\alpha})(\vec{r})=-\Delta_{\mathbb{R}^{n+1}}^{1} \omega_{k}(\widetilde{\alpha})=2 \frac{1-2 k-n}{2-k-n} d Q_{1}
$$

et la Proposition 3.11 permet de conclure.

Si $\widetilde{\alpha} \in \mathcal{S}^{1} \widetilde{H}_{k}$ et si $\alpha$ est sa restriction à $S^{n}$, la décomposition de $\alpha$ selon $\left(H_{3}\right)$ est donnée par

$$
\alpha=\omega_{k}(\alpha)+\frac{1}{k+1} d Q_{0}+\frac{1}{2-k-n} d Q_{1}
$$

En plus, $\omega_{k}(\alpha), d Q_{0}$ et $d Q_{1}$ sont des vecteurs propres de $\Delta_{S^{n}}^{1}$.

On notera

$$
\begin{aligned}
\lambda_{k}^{0} & =k(k+n-1), \\
\lambda_{k}^{1} & =k(k+n-1)+n-2, \\
E_{\lambda_{k}^{0}} & =\left\{d f / f=F / S^{n} \quad \text { et } \quad F \in \widetilde{H}_{k}\right\}, \\
E_{\lambda_{k}^{1}} & =\left\{\omega_{k}(\alpha) / \widetilde{\alpha} \in \mathcal{S}^{1} \widetilde{H}_{k}\right\}, \\
\mathcal{P}^{1}\left(S^{n}, \operatorname{can}\right) & =\left(\bigoplus_{k=1}^{\infty} E_{\lambda_{k}^{0}}\right) \bigoplus\left(\bigoplus_{k=1}^{\infty} E_{\lambda_{k}^{1}}\right) .
\end{aligned}
$$

\section{Proposition 3.14.}

$$
\begin{aligned}
& \operatorname{dim} E_{\lambda_{k}^{0}}=\operatorname{dim} \widetilde{H}_{k} \quad \text { pour } \quad k \geq 1, \\
& \operatorname{dim} E_{\lambda_{k}^{1}}=(n+1) \operatorname{dim} \widetilde{H}_{k}-\left(\operatorname{dim} \widetilde{H}_{k+1}+\operatorname{dim} \widetilde{H}_{k-1}\right) .
\end{aligned}
$$


Preuve: La première égalité est triviale.

Soit $\Phi_{k}: \widetilde{H}_{k+1} \times \widetilde{H}_{k-1} \longrightarrow \mathcal{S}^{1} \widetilde{H}_{k}$ définie par

$$
\Phi_{k}\left(Q_{0}, Q_{1}\right)=\frac{1}{k+1} d Q_{0}+\frac{1}{2-k-n} d r^{2} Q_{1}+\frac{1-2 k-n}{2-k-n} Q_{1} i_{\vec{r}} \text { can } .
$$

Il est facile de voir que $\Phi_{k}\left(Q_{0}, Q_{1}\right) \in \mathcal{S}^{1} \widetilde{H}_{k}$.

Soit

$$
\begin{aligned}
\omega_{k}: \mathcal{S}^{1} \widetilde{H}_{k} & \longrightarrow E_{\lambda_{k}^{1}} \\
\widetilde{\alpha} & \mapsto \omega_{k}(\alpha) .
\end{aligned}
$$

Pour avoir la proposition il suffit de montrer que la suite

$$
0 \longrightarrow \widetilde{H}_{k+1} \times \widetilde{H}_{k-1} \longrightarrow \mathcal{S}^{1} \widetilde{H}_{k} \longrightarrow E_{\lambda_{k}^{1}} \longrightarrow 0
$$

est exacte. Ceci découle immédiatement du fait que

$$
\Phi_{k}\left(Q_{0}, Q_{1}\right)(\vec{r})=Q_{0}+r^{2} Q_{1} .
$$

Théorème 3.1. i) $\mathcal{P}^{1}\left(S^{n}\right.$, can) est dense au sens de la convergence uniforme dans $\mathcal{S}^{1} S^{n}$.

ii) Le spectre de $\Delta_{S^{n}}^{1}$ avec $(n>2)$ est donné par

$$
\operatorname{Spec} \Delta_{S^{n}}^{1}=\{k(k+n-1) \quad k \geq 1, k(k+n-1)+n-2 / \quad k \geq 1\} .
$$

Les multiplicités des valeurs propres sont données par

$$
\begin{aligned}
& \operatorname{multp}\left(\lambda_{1}^{0}\right)=n+1, \\
& \operatorname{multp}\left(\lambda_{k}^{0}\right)=\frac{n(n+1) \ldots(n+k-3)(n+k-2)}{k !}(n+2 k-1), \quad k>1, \\
& \operatorname{multp}\left(\lambda_{1}^{1}\right)=\frac{n(n+1)}{2}, \\
& \operatorname{multp}\left(\lambda_{2}^{1}\right)=\frac{(n-1)(n+1)(n+3)}{3}, \\
& \operatorname{multp}\left(\lambda_{k}^{1}\right)=\frac{(n-1) n(n+1) \ldots(n+k-3)}{(k+1) !} k\left(2 k^{2}+3(n-1) k+(n-1)^{2}\right) \\
& \text { pour } k \geq 3 .
\end{aligned}
$$


Preuve: i) Découle du fait que l'espace des polynômes homogènes est dense dans $C^{\infty}\left(S^{n}\right)$ de (12) et (14).

ii) Découle des Propositions 3.12, 3.13 et 3.14. Il reste à calculer $\operatorname{multp}\left(\lambda_{k}^{1}\right)$. On a

$$
\operatorname{dim} \widetilde{H}_{k}=\frac{n(n+1) \ldots(n+k-3)(n+k-2)}{k !}(n+2 k-1) .
$$

On en déduit que

$$
\begin{aligned}
(n+1) \operatorname{dim} \widetilde{H}_{k}-\left(\operatorname{dim} \widetilde{H}_{k+1}+\operatorname{dim} \widetilde{H}_{k-1}\right) & \\
= & \frac{n(n+1) \ldots(n+k-3)}{(k+1) !}(A(n, k)-B(n, k)-C(n, k))
\end{aligned}
$$

avec

$$
\begin{aligned}
& A(n, k)=(n+1)(n+k-2)(k+1)(n+2 k-1), \\
& B(n, k)=(n+k-2)(n+k-1)(n+2 k+1), \\
& C(n, k)=(n+2 k-3) k(k+1) .
\end{aligned}
$$

Un calcul simple donnerait

$$
\begin{aligned}
A(n, k)= & 2(n+1) k^{3}+3(n+1)(n-1) k^{2} \\
& +(n+1)\left(n^{2}-3\right) k+(n-1)(n-2)(n+1), \\
B(n, k)= & 2 k^{3}+5(n-1) k^{2}+\left(4 n^{2}-7 n+1\right) k+(n+1)(n-2)(n-1), \\
C(n, k)= & 2 k^{3}+(n-1) k^{2}+(n-3) k .
\end{aligned}
$$

Ceci permet de conclure.

Remarque. Spec $\Delta_{S^{2}}^{1}=\{k(k+1) \quad k \geq 1\}$, et $\operatorname{multp}(k(k+1))=$ $2(2 k+1)$.

Proposition 3.15. On a

i) \# $E_{\lambda_{1}^{1}}$ est l'algèbre des champs de Killing de ( $S^{n}$, can).

ii) $\bigoplus_{k=1}^{\infty} E_{\lambda_{k}^{1}}$ est dense au sens de la convergence uniforme dans $\operatorname{Ker} \delta_{0}$. 
Preuve: i) Soit $X$ un champ de Killing de $\left(S^{n}\right.$, can) et soit $\alpha=\omega^{b}(X)$. On a, d'après $(1), \delta_{1}^{*}(\alpha)=0$. D'un autre côté, un calcul simple donne que

$$
\delta_{0}(\alpha)=-\frac{1}{2} \operatorname{Tr} \delta_{1}^{*}(\alpha)=0 .
$$

Donc d'après (5), on aura

$$
\Delta_{S^{n}}^{1}(\alpha)=2(n-1) \alpha .
$$

Or $\lambda_{1}^{1}=2(n-1)$ et donc, si $\mathcal{G}_{1}$ désigne l'algèbre des champs de Killing de $\left(S^{n}\right.$, can $), \omega^{b}\left(\mathcal{G}_{1}\right)$ est contenu dans $E_{\lambda_{1}^{1}}$ et, puisque ils ont la même dimension, ils sont égales.

ii) Découle de $\left(H_{3}\right)$ et de la Proposition 3.13.

\subsection{Spectre et sous-espaces propres de $\Delta_{S^{n}}^{2}$.}

Comme pour $\Delta_{S^{n}}^{1}$, notre calcul sera guidé par les deux décompositions $\left(H_{1}\right)$ et $\left(H_{2}\right)$ données dans l'introduction et que nous rappelons içi.

$\left(H_{1}\right)$

$$
\mathcal{S}^{2} S^{n}=\operatorname{Ker} \delta_{1} \bigoplus \delta_{1}^{*}\left(\Omega^{1}\left(S^{n}\right)\right)
$$

$\left(H_{2}\right) \quad \mathcal{S}^{2} S^{n}=\operatorname{Ker} \delta_{1} \cap \operatorname{Tr}^{-1}(0) \bigoplus\left(\delta_{1}^{*}\left(\Omega^{1}\left(S^{n}\right)\right)+C^{\infty}\left(S^{n}\right) \operatorname{can}\right)$.

Proposition 3.16. Soit $H \in \mathcal{S}^{2} \mathbb{R}^{n+1}$. On a

$$
\begin{aligned}
L_{N} H= & N . H+\frac{2}{r} H-2\left(i_{N} H \odot i_{N} \text { can }\right), \\
L_{N} \circ L_{N} H= & N . N . H+\frac{4}{r} N . H+\frac{2}{r^{2}} H-\frac{2}{r} N .\left(i_{N} H \odot i_{N} \text { can }\right) \\
& -\frac{4}{r}\left(i_{N} H \odot i_{N} \operatorname{can}\right)-2\left(i_{N} L_{N} H \odot i_{N} \text { can }\right) .
\end{aligned}
$$

Preuve:

$L_{N} H\left(\frac{\partial}{\partial x_{i}}, \frac{\partial}{\partial x_{j}}\right)=N \cdot H_{i j}-H\left(\left[N, \frac{\partial}{\partial x_{i}}\right], \frac{\partial}{\partial x_{j}}\right)-H\left(\left[N, \frac{\partial}{\partial x_{j}}\right], \frac{\partial}{\partial x_{i}}\right)$.

Or, d'après (3)

$$
\left[N, \frac{\partial}{\partial x_{i}}\right]=-\widetilde{D}_{\frac{\partial}{\partial x_{i}}} N=-\frac{1}{r}\left(\frac{\partial}{\partial x_{i}}-\left\langle N, \frac{\partial}{\partial x_{i}}\right\rangle N\right) .
$$

Ce qui permet d'avoir la première formule. La deuxième est une application de la première. 
Proposition 3.17. Soit $H \in \mathcal{S}^{2} \widetilde{P}_{k}$ et soit $h$ sa restriction à $S^{n}$. On a, en restriction $\grave{a} S^{n}$,

$$
\begin{aligned}
\Delta_{\mathbb{R}^{n+1}}^{2} H=\Delta_{S^{n}}^{2} h-(k(k+n & -1)+2(n-1)) h \\
& -2 \delta_{1}^{*}\left(i_{\vec{r}} H\right)-2 H(\vec{r}, \vec{r}) \text { can }+2 T r h \text { can } .
\end{aligned}
$$

Preuve: D'après la Proposition 3.16, on a, en restriction à $S^{n}$,

$$
\begin{gathered}
L_{N} H=(k+2) h, \\
L_{N} \circ L_{N} H=(k(k+3)+2) h .
\end{gathered}
$$

La Proposition 3.7 permet alors de conclure.

Proposition 3.18. Soit $F \in \widetilde{H}_{k}$, soit $f$ sa restriction à $S^{n}$ et soit $\widetilde{\alpha} \in \mathcal{S}^{1} \widetilde{H}_{k}$. On a

$$
\begin{aligned}
\Delta_{S^{n}}^{2}(f \text { can }) & =k(k+n-1) f \text { can, } \\
\Delta_{S^{n}}^{2} \delta_{1}^{*}(d f) & =k(k+n-1) \delta_{1}^{*}(d f), \\
\Delta_{S^{n}}^{2} \delta_{1}^{*}\left(\omega_{k}(\alpha)\right) & =(k(k+n-1)+n-2) \delta_{1}^{*}\left(\omega_{k}(\alpha)\right) .
\end{aligned}
$$

Preuve: Ces égalités découlent du Théorème 2.1 et des Propositions $3.9,3.12$ et 3.13 .

Cette proposition nous donne le spectre et les sous-espaces propres de $\Delta_{S^{n}}^{2}$ en restriction à $\operatorname{Im} \delta_{1}^{*}+C^{\infty}\left(S^{n}\right)$ can. Dans ce qui suit, on va donner le spectre et les sous-espaces propres de $\Delta_{S^{n}}^{2}$ en restriction à $\operatorname{Ker} \delta_{1} \cap \operatorname{Tr}^{-1}(0)$ et ce en vertu de $\left(H_{2}\right)$.

Soit $H \in \mathcal{S}^{2} \widetilde{H}_{k}$ et soit $h$ sa restriction à $S^{n}$. Comme pour le calcul du spectre de $\Delta_{S^{n}}^{1}, h$ n'est pas un vecteur propre de $\Delta_{S^{n}}^{2}$. On se propose, dans ce qui suit, de décomposer $h$ suivant $\left(H_{2}\right)$ et trouver un vecteur propre de $\Delta_{S^{n}}^{2}$ pour la valeur propre $\lambda_{k}^{2}=k(k+n-1)+2(n-1)$.

Pour cela, si $H \in \mathcal{S}^{2} \widetilde{P}_{k}$ et si $h$ est sa restriction à $S^{n}$, on pose

$$
\Phi(H)=\Delta_{\mathbb{R}^{n+1}}^{2} H+2 \delta_{1}^{*}\left(i_{\vec{r}} H\right)+2 H(\vec{r}, \vec{r}) \text { can }-2 T r h \text { can } .
$$

$\phi(H)$ est une 2-forme symétrique sur $S^{n}$.

Soit $H \in \mathcal{S}^{2} \widetilde{H}_{k}$ et soit $h$ sa restriction à $S^{n}$. On a

$$
i_{\vec{r}} H=\sum_{i=1}^{n+1} \sum_{j=1}^{n+1} H_{i j} x_{j} d x_{i} .
$$


Or, d'aprés la Proposition 3.8, on a, pour tout $i=1 \ldots n+1$

$$
\sum_{j=1}^{n+1} H_{i j} x_{j}=\alpha_{i}^{0}+r^{2} \alpha_{i}^{1}
$$

avec $\alpha_{i}^{0} \in \widetilde{H}_{k+1}$ et $\alpha_{i}^{1} \in \widetilde{H}_{k-1}$.

On pose

$$
\alpha_{0}=\sum_{i=1}^{n+1} \alpha_{i}^{0} d x_{i}, \quad \text { et } \quad \alpha_{1}=\sum_{i=1}^{n+1} \alpha_{i}^{1} d x_{i} .
$$

Toujours d'après la Proposition 3.8, on a

$$
\alpha_{0}(\vec{r})=P_{0}^{0}+r^{2} P_{1}^{0} \quad \text { et } \quad \alpha_{1}(\vec{r})=P_{0}^{1}+r^{2} P_{1}^{1},
$$

avec $P_{0}^{0} \in \widetilde{H}_{k+2}, P_{1}^{0} \in \widetilde{H}_{k}, P_{0}^{1} \in \widetilde{H}_{k}$ et $P_{1}^{1} \in \widetilde{H}_{k-2}$.

On a, d'après (13),

$$
\begin{aligned}
i_{\vec{r}} H= & \alpha_{0}+r^{2} \alpha_{1} \\
= & \omega_{k+1}\left(\alpha_{0}\right)+r^{2} \omega_{k-1}\left(\alpha_{1}\right) \\
& +\frac{1}{k+2} d P_{0}^{0}+\frac{1}{1-k-n} d r^{2} P_{1}^{0}+\frac{r^{2}}{k} d P_{0}^{1}+\frac{r^{2}}{3-k-n} d r^{2} P_{1}^{1}, \\
H(\vec{r}, \vec{r})= & P_{0}^{0}+r^{2}\left(P_{1}^{0}+P_{0}^{1}\right)+r^{4} P_{1}^{1} .
\end{aligned}
$$

En remarquant que, en restriction à $S^{n}, \operatorname{Tr} H=\operatorname{Tr} h+H(\vec{r}, \vec{r})$, on obtient que

$$
\begin{aligned}
\Phi(H)= & 2 \delta_{1}^{*}\left(\omega_{k+1}\left(\alpha_{0}\right)\right)+2 \delta_{1}^{*}\left(\omega_{k-1}\left(\alpha_{1}\right)\right) \\
& +\frac{2}{k+2} \delta_{1}^{*}\left(d P_{0}^{0}\right)+\frac{2}{1-k-n} \delta_{1}^{*}\left(d r^{2} P_{1}^{0}\right) \\
& +\frac{2}{k} \delta_{1}^{*}\left(d P_{0}^{1}\right)+\frac{2}{3-k-n} \delta_{1}^{*}\left(d r^{2} P_{1}^{1}\right) \\
& +4\left(P_{0}^{0}+P_{1}^{0}+P_{0}^{1}+P_{1}^{1}\right) \text { can }-2 T r H \text { can } .
\end{aligned}
$$


On pose, maintenant

$$
\begin{aligned}
& \Omega_{k}(H)=h-\frac{1}{k} \delta_{1}^{*}\left(\omega_{k+1}\left(\alpha_{0}\right)\right)+\frac{1}{k+n-1} \delta_{1}^{*}\left(\omega_{k-1}\left(\alpha_{1}\right)\right) \\
& -\frac{1}{2(k+1)(k+2)} \delta_{1}^{*}\left(d P_{0}^{0}\right)+\frac{1}{(n-1)(1-k-n)} \delta_{1}^{*}\left(d P_{1}^{0}\right) \\
& +\frac{1}{(n-1) k} \delta_{1}^{*}\left(d P_{0}^{1}\right)+\frac{1}{2(k+n-2)(3-k-n)} \delta_{1}^{*}\left(d P_{1}^{1}\right) \\
& +\left(-\frac{1}{k+1} P_{0}^{0}+\frac{2}{n-1}\left(P_{1}^{0}+P_{0}^{1}\right)\right. \\
& \left.+\frac{1}{k+n-2} P_{1}^{1}+\frac{1}{1-n} \operatorname{Tr} H\right) \text { can, } \\
& \widetilde{\Omega}_{k}(H)=H-\frac{1}{k} \widetilde{\delta}_{1}^{*}\left(\omega_{k+1}\left(\alpha_{0}\right)\right)+\frac{r^{2}}{k+n-1} \widetilde{\delta}_{1}^{*}\left(\omega_{k-1}\left(\alpha_{1}\right)\right) \\
& -\frac{1}{2(k+1)(k+2)} \widetilde{\delta}_{1}^{*}\left(d P_{0}^{0}\right)+\frac{r^{2}}{(n-1)(1-k-n)} \widetilde{\delta}_{1}^{*}\left(d P_{1}^{0}\right) \\
& +\frac{r^{2}}{k(n-1)} \widetilde{\delta}_{1}^{*}\left(d P_{0}^{1}\right)+\frac{r^{4}}{2(k+n-2)(3-k-n)} \widetilde{\delta}_{1}^{*}\left(d P_{1}^{1}\right) \\
& -2 \frac{k^{2}+3(n-1) k+\left(n^{2}-1\right)}{k(n-1)(1-k-n)} P_{1}^{0} \operatorname{can}+\frac{1}{1-n} \operatorname{Tr} H \text { can } \\
& +\frac{2 k^{2}+(3 n-7) k+n^{2}-4 n+7}{(3-k-n)(k+n-1)(k+n-2)} r^{2} P_{1}^{1} \text { can . }
\end{aligned}
$$

Un calcul direct utilisant la Proposition 3.3 permet de vérifier que $\widetilde{\Omega}_{k}(H) \in \mathcal{S}^{2} \widetilde{P}_{k}$ et que sa restriction à $S^{n}$ est exactement $\Omega_{k}(H)$.

Proposition 3.19. Soit $H \in \mathcal{S}^{2} \widetilde{H}_{k}$. On a

$$
\Delta_{S^{n}}^{2} \Omega_{k}(H)=(k(k+n-1)+2(n-1)) \Omega_{k}(H) .
$$

Preuve: D'après la Proposition 3.17, il suffit de vérifier que $\Phi\left(\tilde{\Omega}_{k}(H)\right)=0$. 
Or $\Omega_{k}(H)-h$, restriction de $\tilde{\Omega}_{k}(H)-H$, est la somme de vecteurs propres de $\Delta_{S^{n}}^{2}$ dont les coefficients on été déterminée pour que justement $\Phi\left(\tilde{\Omega}_{k}(H)\right)$ soit égale à 0 . La vérification peut se faire, en remarquant que, si $\phi$ est un vecteur propre de $\Delta_{S^{n}}^{2}$ pour la valeur propre $\lambda$ et $\widetilde{\phi}$ est une 2 -forme homogène de degré $k$ qui prolonge $\phi$, on a, en vertu de la Proposition 3.17 , que

$$
\Phi(\widetilde{\phi})=(\lambda-(k(k+n-1)+2(n-1))) \phi
$$

Proposition 3.20. Soit $H \in \mathcal{S}^{2} \widetilde{H}_{k}$. On a

$$
\operatorname{Tr} \Omega_{k}(H)=0 \quad \text { et } \quad \delta_{1}\left(\Omega_{k}(H)\right)=0 .
$$

Preuve: D'après la proposition précédente, $\Omega_{k}(H)$ est un vecteur propre de $\Delta_{S^{n}}^{2}$ pour la valeur propre $k(k+n-1)+2(n-1)$ et donc, d'après la Proposition 3.18, on a

$$
\left\langle\Omega_{k}(H), f \text { can }\right\rangle=\left\langle\Omega_{k}(H), \delta_{1}^{*}(d f)\right\rangle=\left\langle\Omega_{k}(H), \delta_{1}^{*}\left(\omega_{k}(\alpha)\right)\right\rangle=0
$$

pour tout $f \in \widetilde{H}_{k}$ et tout $\widetilde{\alpha} \in \mathcal{S}^{1} \widetilde{H}_{k}$. Par un argument de densité, on déduit que $\Omega_{k}(H)$ est orthogonal à $C^{\infty}\left(S^{n}\right)$ can et à $\delta_{1}^{*}\left(\mathcal{S}^{1} S^{n}\right)$, ce qui prouve la proposition.

Soit $H \in \mathcal{S}^{2} \widetilde{H}_{k}$ et si $h$ est sa restriction à $S^{n}$, la décomposition de $h$ suivant $\left(H_{2}\right)$ est donnée par

$$
\begin{aligned}
h= & \Omega_{k}(H)+\frac{1}{k} \delta_{1}^{*}\left(\omega_{k+1}\left(\alpha_{0}\right)\right)-\frac{1}{k+n-1} \delta_{1}^{*}\left(\omega_{k-1}\left(\alpha_{1}\right)\right) \\
& +\frac{1}{2(k+1)(k+2)} \delta_{1}^{*}\left(d P_{0}^{0}\right)-\frac{1}{(n-1)(1-k-n)} \delta_{1}^{*}\left(d P_{1}^{0}\right) \\
& -\frac{1}{(n-1) k} \delta_{1}^{*}\left(d P_{0}^{1}\right)-\frac{1}{2(k+n-2)(3-k-n)} \delta_{1}^{*}\left(d P_{1}^{1}\right) \\
& +\left(+\frac{1}{k+1} P_{0}^{0}-\frac{2}{n-1}\left(P_{1}^{0}+P_{0}^{1}\right)-\frac{1}{k+n-2} P_{1}^{1}-\frac{1}{1-n} \operatorname{Tr} H\right) \operatorname{can} .
\end{aligned}
$$


D'un autre côté et puisque $\operatorname{Tr} \Omega_{k}(H)=0, \operatorname{Tr} H$ ne dépend que de $\alpha_{0}$ et $\alpha_{1}$. Pour calculer la multiplicité des valeurs propres, on définit alors

$$
\begin{aligned}
\Psi_{k}\left(\alpha_{0}, \alpha_{1}\right)= & -\frac{1}{k} \widetilde{\delta}_{1}^{*}\left(\omega_{k+1}\left(\alpha_{0}\right)\right)+\frac{r^{2}}{k+n-1} \widetilde{\delta}_{1}^{*}\left(\omega_{k-1}\left(\alpha_{1}\right)\right) \\
& -\frac{1}{2(k+1)(k+2)} \widetilde{\delta}_{1}^{*}\left(d P_{0}^{0}\right)+\frac{r^{2}}{(n-1)(1-k-n)} \widetilde{\delta}_{1}^{*}\left(d P_{1}^{0}\right) \\
& +\frac{r^{2}}{k(n-1)} \widetilde{\delta}_{1}^{*}\left(d P_{0}^{1}\right)+\frac{r^{4}}{2(k+n-1)(3-k-n)} \widetilde{\delta}_{1}^{*}\left(d P_{1}^{1}\right) \\
& -2 \frac{k^{2}+3(n-1) k+\left(n^{2}-1\right)}{k(n-1)(1-k-n)} P_{1}^{0} \operatorname{can}+\frac{1}{1-n} \operatorname{Tr} H \mathrm{can} \\
& +\frac{2 k^{2}+(3 n-7) k+n^{2}-4 n+7}{(3-k-n)(k+n-1)(k+n-2)} r^{2} P_{1}^{1} \operatorname{can} .
\end{aligned}
$$

On notera

$$
\begin{aligned}
\lambda_{k}^{0} & =k(k+n-1), \\
\lambda_{k}^{1} & =k(k+n-1)+n-2, \\
\lambda_{k}^{2} & =k(k+n-1)+2(n-1), \\
G_{\lambda_{k}^{0}} & =\left\{f \operatorname{can}+\delta_{1}^{*}(d q) / f=F / S^{n}, \quad q=Q / S^{n} \quad \text { et } \quad F, Q \in \widetilde{H}_{k}\right\}, \\
G_{\lambda_{k}^{1}} & =\left\{\delta_{1}^{*}\left(\omega_{k}(\alpha)\right) / \widetilde{\alpha} \in \mathcal{S}^{1} \widetilde{H}_{k}\right\}, \\
G_{\lambda_{k}^{2}} & =\left\{\Omega_{k}(H) / H \in \mathcal{S}^{2} \widetilde{H}_{k}\right\}, \\
\mathcal{P}^{2}\left(S^{n}, \operatorname{can}\right) & =\left(\bigoplus_{k=0}^{\infty} G_{\lambda_{k}^{0}}\right) \bigoplus\left(\bigoplus_{k=2}^{\infty} G_{\lambda_{k}^{1}}\right) \bigoplus\left(\bigoplus_{k=2}^{\infty} G_{\lambda_{k}^{2}}\right) .
\end{aligned}
$$

Proposition 3.21. On a

$\operatorname{dim} G_{\lambda_{0}^{0}}=1$,

$\operatorname{dim} G_{\lambda_{1}^{0}}=n+1$

$\operatorname{dim} G_{\lambda_{k}^{0}}=2 \operatorname{dim} \widetilde{H}_{k} \quad$ pour $\quad k \geq 2$,

$\operatorname{dim} G_{\lambda_{k}^{1}}=(n+1) \operatorname{dim} \widetilde{H}_{k}-\left(\operatorname{dim} \widetilde{H}_{k+1}+\operatorname{dim} \widetilde{H}_{k-1}\right) \quad$ pour $\quad k \geq 2$,

$\operatorname{dim} G_{\lambda_{k}^{2}}=\frac{1}{2}(n+2)(n+1) \operatorname{dim} \widetilde{H}_{k}-(n+1)\left(\operatorname{dim} \widetilde{H}_{k+1}+\operatorname{dim} \widetilde{H}_{k-1}\right)$. 
Preuve: Les deux premières égalités sont triviales. Montrons la troisième. Soient $F, Q \in \widetilde{H}_{k}$ et soient $f, q$ leurs restrictions à $S^{n}$. Supposons que

$$
f \operatorname{can}+\delta_{1}^{*}(d q)=0 .
$$

D'après (7) et (19), on a

$$
\begin{aligned}
\delta_{1}(f \text { can }) & =-d f \\
\delta_{1} \delta_{1}^{*}(d q) & =2(k(k+n-1)-(n-1)) d q .
\end{aligned}
$$

On en déduit que $f=2(k(k+n-1)-(n-1)) q$ et par homogénéité $F=2(k(k+n-1)-(n-1)) Q$.

D'un autre côté, un calcul direct donne

$$
\operatorname{Tr} \delta_{1}^{*}(d q)=-2 \delta_{0}(d q)=-2 k(k+n-1) q
$$

et en prenant la trace dans $(*)$, on déduit que $q=0$ et par homogénéité on aura $F=Q=0$. Ceci prouve la troisième égalité.

Soit $\Phi_{k}: \mathcal{S}^{1} \widetilde{H}_{k+1} \times \mathcal{S}^{1} \widetilde{H}_{k-1} \longrightarrow \mathcal{S}^{2} \widetilde{H}_{k}$ définie par

$$
\Phi_{k}\left(\alpha_{0}, \alpha_{1}\right)=-\Psi_{k}\left(\alpha_{0}, \alpha_{1}\right)+\beta \odot i_{\vec{r}} \text { can },
$$

où $\beta$ est définie de manière unique pour que $i_{\vec{r}} \Phi_{k}\left(\alpha_{0}, \alpha_{1}\right)=\alpha_{0}+r^{2} \alpha_{1}$. Et soit

$$
\begin{aligned}
\Omega_{k}: \mathcal{S}^{2} \widetilde{H}_{k} & \longrightarrow G_{\lambda_{k}^{2}} \\
H & \mapsto \Omega_{k}(H) .
\end{aligned}
$$

Pour avoir la proposition il suffit de montrer que la suite

$$
0 \longrightarrow \mathcal{S}^{1} \widetilde{H}_{k+1} \times \mathcal{S}^{1} \widetilde{H}_{k-1} \longrightarrow \mathcal{S}^{2} \widetilde{H}_{k} \longrightarrow G_{\lambda_{k}^{2}} \longrightarrow 0
$$

est exacte. Ce qui est très simple à vérifier. 
Théorème 3.2. i) $\mathcal{P}^{2}\left(S^{n}\right.$, can) est dense au sens de la convergence uniforme dans $\mathcal{S}^{2} S^{n}$.

ii) Le spectre de $\Delta_{S^{n}}^{2}(n>2)$ est donné par

$$
\begin{array}{r}
\operatorname{Spec} \Delta_{S^{n}}^{2}=\{k(k+n-1) \quad k \geq 0, k(k+n-1)+n-2 \quad k \geq 2, \\
k(k+n-1)+2(n-1) \quad k \geq 2\} .
\end{array}
$$

Les multiplicités des valeurs propres sont données par

$$
\begin{aligned}
\operatorname{multp}\left(\lambda_{0}^{0}\right)= & 1, \\
\operatorname{multp}\left(\lambda_{1}^{0}\right)= & n+1, \\
\operatorname{multp}\left(\lambda_{k}^{0}\right)= & 2 \frac{n(n+1) \ldots(n+k-3)(n+k-2)}{k !}(n+2 k-1), \quad k \geq 2, \\
\operatorname{multp}\left(\lambda_{2}^{1}\right)= & \frac{(n-1)(n+1)(n+3)}{3}, \\
\operatorname{multp}\left(\lambda_{k}^{1}\right)= & \frac{(n-1) n(n+1) \ldots(n+k-3)}{(k+1) !} k \\
& \times\left(2 k^{2}+3(n-1) k+(n-1)^{2}\right) \quad k \geq 3, \\
\operatorname{multp}\left(\lambda_{2}^{2}\right)= & \frac{(n+1)(n+2)(n+3)(n-2)}{12}, \\
\operatorname{multp}\left(\lambda_{k}^{2}\right)= & \frac{1}{2} \frac{n(n+1) \ldots(n+k-3)}{(k+1) !}(n+1)(n-2) \\
& \times\left(2 k^{3}+3(n-1) k^{2}+\left(n^{2}-4 n+1\right) k-n(n-1)\right) \quad k \geq 3 .
\end{aligned}
$$

Preuve: i) Découle du fait que l'espace des polynômes homogènes est dense dans $C^{\infty}\left(S^{n}\right)$ de $(12)$ et $(16)$.

ii) Découle des Propositions $3.18,3.19$ et 3.21 . Il reste à vérifier $\operatorname{multp}\left(\lambda_{k}^{1}\right)$ et $\operatorname{multp}\left(\lambda_{k}^{2}\right)$.

La relation (1), la Proposition 3.15 et le Théorème 3.1 permettent de donner la multiplicité de $\operatorname{multp}\left(\lambda_{k}^{1}\right)$.

$$
\operatorname{dim} \widetilde{H}_{k}=\frac{n(n+1) \ldots(n+k-3)(n+k-2)}{k !}(n+2 k-1) .
$$


On en déduit que

$$
\begin{aligned}
& \frac{1}{2}(n+2)(n+1) \operatorname{dim} \widetilde{H}_{k}-(n+1)\left(\operatorname{dim} \widetilde{H}_{k+1}+\operatorname{dim} \widetilde{H}_{k-1}\right) \\
& =\frac{(n+1)}{2} \frac{n(n+1) \ldots(n+k-3)}{(k+1) !}\left(\frac{n+2}{n+1} A(n, k)-2 B(n, k)-2 C(n, k)\right),
\end{aligned}
$$

avec

$$
\begin{aligned}
& A(n, k)=(n+1)(n+k-2)(k+1)(n+2 k-1), \\
& B(n, k)=(n+k-2)(n+k-1)(n+2 k+1), \\
& C(n, k)=(n+2 k-3) k(k+1) .
\end{aligned}
$$

Avec (voir la preuve du Théorème 3.1)

$$
\begin{aligned}
A(n, k)= & 2(n+1) k^{3}+3(n+1)(n-1) k^{2} \\
& +(n+1)\left(n^{2}-3\right) k+(n-1)(n-2)(n+1), \\
B(n, k)= & 2 k^{3}+5(n-1) k^{2}+\left(4 n^{2}-7 n+1\right) k+(n+1)(n-2)(n-1), \\
C(n, k)= & 2 k^{3}+(n-1) k^{2}+(n-3) k .
\end{aligned}
$$

Ceci permet de donner la multiplicité de $\operatorname{multp}\left(\lambda_{k}^{2}\right)$.

Remarque. Spec $\Delta_{S^{2}}^{2}=\{k(k+1) \quad k \geq 1\}$, et $\operatorname{multp}(k(k+1))=$ $3(2 k+1)$.

Soit $F \in \widetilde{\mathcal{H}}_{k}$ et soit $f$ sa restriction à $S^{n}$. On pose

$$
N(f)=\delta_{1}^{*}(d f)+2(k(k+n-1)-(n-1)) f \text { can } .
$$

Proposition 3.22. Soit $F \in \widetilde{\mathcal{H}}_{k}$ et soit $f$ sa restriction $\grave{a} S^{n}$. On a

$$
\delta_{1}(N(f))=0
$$

Preuve: D'après la Proposition 3.12, on a

$$
\Delta_{S^{n}}^{1} d f=k(k+n-1) d f .
$$

Soit, en vertu de (5),

$$
\delta_{1} \delta_{1}^{*}(d f)=(k(k+n-1)-2(n-1)) d f+d \delta_{0} d f .
$$


Or, d'après la Proposition $3.9, \delta_{0} d f=k(k+n-1) f$. On obtient donc que

$$
\delta_{1} \delta_{1}^{*}(d f)=2(k(k+n-1)-(n-1)) d f .
$$

On peut conclure, en remarquant que $\delta_{1}(f$ can $)=-d f$.

On note

$$
\begin{aligned}
& N\left(\widetilde{\mathcal{H}}_{k}\right)=\left\{\delta_{1}^{*}(d f)+2(k(k+n-1)-(n-1)) f\right. \text { can } \\
&\left.\qquad f=F / S^{n} \quad \text { et } \quad F \in \widetilde{\mathcal{H}}_{k}\right\}, \\
& N=\bigoplus_{k=0}^{\infty} N\left(\widetilde{\mathcal{H}}_{k}\right) .
\end{aligned}
$$

Comme annoncé dans l'introduction, on obtient:

Théorème 3.3. i) $N \oplus\left(\bigoplus_{k=2}^{\infty} G_{\lambda_{k}^{2}}\right)$ est dense au sens de la convergence uniforme dans $\operatorname{Ker} \delta_{1}$.

ii) $\left(\bigoplus_{k=2}^{\infty} G_{\lambda_{k}^{2}}\right)$ est dense au sens de la convergence uniforme dans ker $\delta_{1} \cap \operatorname{Tr}^{-1}(0)$.

Preuve: D'après les Propositions 3.22 et 3.20 , on a

$$
N \bigoplus\left(\bigoplus_{k=2}^{\infty} G_{\lambda_{k}^{2}}\right) \subset \operatorname{Ker} \delta_{1}, \quad\left(\bigoplus_{k=2}^{\infty} G_{\lambda_{k}^{2}}\right) \subset \operatorname{Ker} \delta_{1} \cap \operatorname{Tr}^{-1}(0)
$$

i) et ii) découlent alors des décomposition $\left(H_{1}\right)$ et $\left(H_{2}\right)$.

Pour finir cette section, on va donner deux applications du Théorème 2.2 pour retrouver deux résultats bien connus.

Théorème 3.4 [Be-Eb]. Pour toute 2-forme symétrique $h$ sur $S^{2}$, il existe un couple $(f, X)$ où $f$ est une fonction différentiable et $X$ un champ de vecteurs, tels que

$$
h=L_{X} \operatorname{can}+f \text { can } .
$$

Preuve: Découle de i) du Théorème 3.2 et du fait que, pour $n=2$, $\operatorname{dim} G_{\lambda_{k}^{2}}=0$. 
Proposition 3.23. La structure d'Einstein canonique sur $S^{n}$ est rigide.

Preuve: Soit $h$ une déformation d'Einstein infinitésimale. D'après [Be2, p. 347] et un calcul simple, $h$ vérifie

$$
\operatorname{Tr} h=0, \delta_{1}(h)=0 \quad \text { et } \quad \Delta_{S^{n}}^{2}(h)=4(n-1) h .
$$

D'après le Théorème $3.2 h=0$. $\mathbf{a}$ (Voir [Be2, p. 355] pour plus de détails.)

\section{Laplaciens de Lichnerowicz sur les projectifs réels}

On notera $P: S^{n} \longrightarrow \mathbb{R} P^{n}$ le revêtement canonique du projectif réel de dimension $n$ par la sphère $S^{n}$.

Proposition 4.1. Soit $f \in C^{\infty}\left(\mathbb{R} P^{n}\right), \alpha \in \mathcal{S}^{1} \mathbb{R} P^{n}$ et $h \in \mathcal{S}^{2} \mathbb{R} P^{n}$. On $a$

$$
\begin{aligned}
& \Delta_{S^{n}}^{0}\left(P^{*} f\right)=P^{*} \Delta_{\mathbb{R} P^{n}}^{0}(f), \\
& \Delta_{S^{n}}^{1}\left(P^{*} \alpha\right)=P^{*} \Delta_{\mathbb{R} P^{n}}^{1}(\alpha), \\
& \Delta_{S^{n}}^{2}\left(P^{*} h\right)=P^{*} \Delta_{\mathbb{R} P^{n}}^{2}(h) .
\end{aligned}
$$

Preuve: Pour la première relation voir [Be-Ga-Ma, p. 129]. Les deux dernières découlent du fait que le revêtement $P: S^{n} \longrightarrow \mathbb{R} P^{n}$ est une isométrie locale.

On identifiera $C^{\infty}\left(\mathbb{R} P^{n}\right)$ au sous-espace vectoriel de $C^{\infty}\left(S^{n}\right)$ des fonctions paire pour l'antipodie, $\mathcal{S}^{1} \mathbb{R} P^{n}$ au sous-espace vectoriel de $\mathcal{S}^{1} S^{n}$ des 1 -formes invariante par l'antipodie et $\mathcal{S}^{2} \mathbb{R} P^{n}$ au sous-espace vectoriel des 2 -formes symétriques invariantes par antipodie.

Les notations sont celles la section précédente. On notera

$$
\begin{aligned}
\mathcal{P}^{1}\left(\mathbb{R} P^{n}, \text { can }\right) & =\left(\bigoplus_{k=1}^{\infty} E_{\lambda_{2 k}^{0}}\right) \bigoplus\left(\bigoplus_{k=0}^{\infty} E_{\lambda_{2 k+1}^{1}}\right) \\
\mathcal{P}^{2}\left(\mathbb{R} P^{n}, \text { can }\right) & =\left(\bigoplus_{k=0}^{\infty} G_{\lambda_{2 k}^{0}}\right) \bigoplus\left(\bigoplus_{k=2}^{\infty} G_{\lambda_{2 k+1}^{1}}\right) \bigoplus\left(\bigoplus_{k=1}^{\infty} G_{\lambda_{2 k}^{2}}\right) \\
N^{+} & =\bigoplus_{k=0}^{\infty} N\left(\widetilde{\mathcal{H}}_{2 k}\right)
\end{aligned}
$$

Les résultats suivants sont une conséquence immédiates des Théorèmes 3.1, 2.2, 2.3, la Proposition 3.15 et la Proposition 4.1. 
Théorème 4.1. i) $\mathcal{P}^{1}\left(\mathbb{R} P^{n}\right.$, can) est dense au sens de la convergence uniforme dans $\mathcal{S}^{1} \mathbb{R} P^{n}$.

ii) Le spectre de $\Delta_{\mathbb{R} P^{n}}^{1}$ avec $(n>2)$ est donné par

$\operatorname{Spec} \Delta_{\mathbb{R} P^{n}}^{1}=\{2 k(2 k+n-1) \quad k \geq 1,(2 k+1)(2 k+n)+n-2 / \quad k \geq 0\}$.

Les multiplicités des valeurs propres sont données par

$$
\begin{aligned}
\operatorname{multp}\left(\lambda_{2 k}^{0}\right)= & \frac{n(n+1) \ldots(n+2 k-3)(n+2 k-2)}{(2 k) !}(n+4 k-1), \quad k \geq 1, \\
\operatorname{multp}\left(\lambda_{1}^{1}\right)= & \frac{n(n+1)}{2}, \\
\operatorname{multp}\left(\lambda_{2 k+1}^{1}\right)= & \frac{(n-1) n(n+1) \ldots(n+2 k-2)}{(2 k+2) !} \\
& \times(2 k+1)\left(8 k^{2}+2(3 n+1) k+(n+1) n\right)
\end{aligned}
$$

pour $k \geq 1$.

Théorème 4.2. i) $\mathcal{P}^{2}\left(\mathbb{R} P^{n}\right.$, can) est dense au sens de la convergence uniforme dans $\mathcal{S}^{2} \mathbb{R} P^{n}$.

ii) Le spectre de $\Delta_{\mathbb{R} P^{n}}^{2}(n>2)$ est donné par

$\operatorname{Spec} \Delta_{\mathbb{R} P^{n}}^{2}=\{2 k(2 k+n-1) \quad k \geq 0,(2 k+1)(2 k+n)+n-2 \quad k \geq 1$,

$$
2 k(2 k+n-1)+2(n-1) \quad k \geq 2\} .
$$

Les multiplicités des valeurs propres sont données par

$$
\begin{aligned}
\operatorname{multp}\left(\lambda_{0}^{0}\right)= & 1 \\
\operatorname{multp}\left(\lambda_{2 k}^{0}\right)= & 2 \frac{n(n+1) \ldots(n+2 k-3)(n+2 k-2)}{(2 k) !} \\
& \times(n+4 k-1), \quad k \geq 1
\end{aligned}
$$

$\operatorname{multp}\left(\lambda_{2 k+1}^{1}\right)=\frac{(n-1) n(n+1) \ldots(n+2 k-2)}{(2 k+2) !}$

$$
\times(2 k+1)\left(8 k^{2}+2(3 n+1) k+(n+1) n\right),
$$

$$
\begin{aligned}
\operatorname{multp}\left(\lambda_{2}^{2}\right)= & \frac{(n+1)(n+2)(n+3)(n-2)}{12}, \\
\operatorname{multp}\left(\lambda_{2 k}^{2}\right)= & \frac{1}{2} \frac{n(n+1) \ldots(n+2 k-3)}{(2 k+1) !}(n+1)(n-2) \\
& \times\left(16 k^{3}+12(n-1) k^{2}+2\left(n^{2}-4 n+1\right) k-n(n-1)\right) \quad k \geq 2 .
\end{aligned}
$$


Proposition 4.2. On a

i) $\# E_{\lambda_{1}^{1}}$ est l'algèbre des champs de Killing de $\left(\mathbb{R} P^{n}\right.$, can).

ii) $\bigoplus_{k=0}^{\infty} E_{\lambda_{2 k+1}^{1}}$ est dense au sens de la convergence uniforme dans Ker $\delta_{0}$.

Théorème 4.3. i) $N^{+} \bigoplus\left(\bigoplus_{k=1}^{\infty} G_{\lambda_{2 k}^{2}}\right)$ est dense au sens de la convergence uniforme dans $\operatorname{Ker} \delta_{1}$.

ii) $\left(\bigoplus_{k=2}^{\infty} G_{\lambda_{2 k}^{2}}\right)$ est dense au sens de la convergence uniforme dans ker $\delta_{1} \cap \operatorname{Tr}^{-1}(0)$.

\section{Références}

[Be1] A. Besse, "Manifolds all of whose geodesics are closed," Springer-Verlag, Berlin-Heidelberg-New York, 1978.

[Be2] A. Besse, "Einstein manifolds," Springer-Verlag, Berlin-Heidelberg-New York, 1987.

[Be-Eb] M. Berger et D. Ebin, Some decomposition of the space of symmetric tensors on riemannian manifold, J. Differential Geom. 3 (1969), 379-392.

[Be-Ga-Ma] M. Berger, P. Gauduchon et E. Mazet, "Le spectre d'une variété riemannienne," Lecture Notes in Math. 194, SpringerVerlag, Berlin-Heidelberg-New York, 1971.

[Be-Mi] B. L. Beers et R. S. Millman, The spectra of the LaplaceBeltrami operator on compact, semisimple Lie groups, Amer. J. Math. 99(4) (1975), 801-807.

[Ga-Me] S. Gallot et D. Meyer, Opérateur de courbure et laplaciens des formes différentielles d'une variété riemannienne, J. Math. Pures Appl. 54 (1975), 259-289.

[Ik-Ta] A. Ikeda ET Y. TANiguchi, Spectra and eigenforms of Laplacian on $S^{n}$ and $P^{n}(\mathbb{C})$, Osaka J. Math. 15(3) (1978), 515-546.

[Iw-Ka] I. Iwasaki et K. Katase, On the spectrum of the Laplace operator on $\bigwedge^{*}\left(S^{n}\right)$, Proc. Japan Acad. Ser. A Math. Sci. 55 (1979), 141-145.

[L] A. Lichnerowicz, Propogateurs et commutateurs en relativité générale, Inst. Hautes Études Sci. Publ. Math. 10 (1961). 
[M] R. Michel, Problèmes d'analyse géométrique liés à la conjecture de Blaschke, Bull. Soc. Math. France 101 (1973), 17-69.

\author{
Faculté des Sciences et Techniques \\ Université Cadi-Ayyad \\ Gueliz, BP 618 \\ Marrakech \\ MAROC \\ e-mail: fstg@cybernet.net.ma
}

Primera versió rebuda el 19 de gener de 1998, darrera versió rebuda el 16 de desembre de 1998 